\title{
Thrombosis and Hemostasis Issues in Cancer Patients with COVID-19
}

\author{
Netanel A. Horowitz, MD ${ }^{1,2}$ Benjamin Brenner, MD ${ }^{1,2,3}$ \\ ${ }^{1}$ Department of Hematology and Bone Marrow Transplantation, \\ Rambam Health Care Campus, Haifa, Israel \\ 2 The Ruth and Bruce Rappaport Faculty of Medicine, Technion - Israel \\ Institute of Technology, Haifa, Israel \\ Address for correspondence Netanel A. Horowitz, MD, Department \\ of Hematology and Bone Marrow Transplantation, Rambam Health \\ Care Campus, 8, Ha'Aliya Street, Haifa 3109601, Israel \\ (e-mail: n_horowitz@rambam.health.gov.il).
}

${ }^{3}$ Department of Obstetrics and Gynaecology, The First I.M. Sechenov Moscow State Medical University, Moscow, Russia

Semin Thromb Hemost 2020;46:785-788.

Coronavirus disease 2019 (COVID-19) is an infectious pandemic disorder caused by severe acute respiratory syndrome coronavirus 2 (SARS-CoV2), a single-stranded RNA $\beta$-coronavirus. Virus particles that can be inhaled through the respiratory system and invade lung alveolar cells may cause a limited viral disease. However, in some patients, severe complications, including systemic inflammatory response syndrome, acute respiratory disease syndrome, multiple organ failure, and shock may develop. These presentations are particularly frequent among several risk groups, including older patients, those with hypertension, obesity, cardiovascular, pulmonary and renal diseases, autoimmunity disturbances, and cancer. COVID-19 is associated with severe thrombotic complications, both micro- and macrovascular, substantially including deep vein thrombosis (DVT), pulmonary embolism (PE), primary pulmonary arterial thrombosis, up to disseminate intravascular coagulation (DIC)-like syndrome. ${ }^{1-4}$

Cancer patients are more vulnerable to COVID-19 infection and their disease course is likely to be more aggressive. A recent report evaluated 1,524 patients, admitted to the Department of Radiation and Medical Oncology of Zhongnan Hospital of Wuhan University. While the rate of COVID-19 was $0.79 \%$ among cancer patients, it was $0.37 \%$ in the general population of Wuhan during the same time period (odds ratio [OR]: 2.31, 95\% confidence interval [CI]: 1.89-3.02). Patients with non-small-cell lung cancer (NSCLC) displayed higher incidence of COVID-19, especially those $>60$ years of age ( 4.3 vs. $1.8 \%$ in those aged $\leq 60$ years with NSCLC). ${ }^{5}$ In another study from China, cancer patients were found to have higher risk of severe events (i.e., death or admission to the intensive care unit [ICU] for invasive ventilation) (7/18 [39\%] vs. $124 / 1,572$ [8\%] patients; $p=0.0003){ }^{6}$

The COVID-19 and Cancer Consortium (CCC19) registry recently reported a large cohort study of 928 patients from the United States, Canada, and Spain. The primary endpoint

was all-cause mortality within 30 days of diagnosis of COVID-19. The median age was 66 years (57-76), and 50\% of patients were males. The leading malignancies were breast (21\%) and prostate (16\%). The ratio of active anticancer treatment or active (measurable) cancer was high, 39 and 43\%, respectively. As per analysis dated May 7, 2020, 121 (13\%) patients died. The independent risk factors associated with 30-day mortality included age (per 10 years; partially adjusted OR: $1.84,95 \% \mathrm{CI}: 1.53-2.21)$, smoking status (1.60, 1.03-2.47), male sex (OR: $1.63,95 \% \mathrm{CI}: 1.07-2.48$ ), number of comorbidities ( 2 vs. none: OR: $4.50,95 \%$ CI: $1.33-15.28$ ), the Eastern Cooperative Oncology Group performance status of 2 or higher (OR: $3.89,95 \%$ CI: 2.11-7.18), active cancer (5.20, 2.77-9.77), and use of azithromycin plus hydroxychloroquine (OR: 2.93, 95\% CI: 1.79-4.79). Of note, ethnicity, obesity, tumor type, and the anticancer therapy applied had no impact on mortality. As for environmental factors, residence in Canada or the U.S.-Midwest were associated with decreased 30-day all-cause mortality compared with residence in the U.S.-Northeast (OR: $0.24,95 \%$ CI: 0.07-0.84 and OR: $0.50,95 \%$ CI: $0.28-0.90$, respectively). ${ }^{7}$

\section{Thrombosis and Cancer}

Venous thromboembolism (VTE) events are common among cancer patients, and their occurrence contributes significantly to morbidity and mortality. ${ }^{8,9}$ The risk of VTE is increased by five- to sevenfold among patients with malignant diseases, while fatal PE is three times more common in this population relative to noncancer patients. Annually, $0.5 \%$ of cancer patients experience thrombosis compared with a $0.1 \%$ incidence rate in general population. Arterial events (i.e., acute myocardial infarctions [MIs] and ischemic strokes) are also more common in cancer patients with solid tumors. In a large retrospective study, including 279,719 pairs of patients with

published online August 12, 2020
Issue Theme Maintaining Hemostasis and Preventing Thrombosis in COVID-19 -Part I; Guest Editors: Emmanuel J. Favaloro, PhD, FFSc (RCPA), and Giuseppi Lippi, MD.
Copyright $\odot 2020$ by Thieme Medical Publishers, Inc., 333 Seventh Avenue, New York, NY 10001, USA. Tel: +1(212) 760-0888.
DOI https://doi.org/ 10.1055/s-0040-1714275. ISSN 0094-6176. 
cancer and matched controls, the 6-month cumulative incidence of arterial thromboembolism was $4.7 \%$ in patients compared with $2.2 \%$ in controls. ${ }^{10}$ In a recent report on 32,141 hematological cancer patients, the 10-year absolute risk of thrombotic complications following cancer diagnosis was 3.3\% for MI, 3.5\% for ischemic stroke, and 5.2\% for VTE. Overall, patients with hematological cancer were at increased risk for MI, ischemic stroke, and VTE compared with general population. ${ }^{11}$ In cancer patients, DIC may present in a chronic form, often exacerbating into a full-blown state following surgery, chemotherapy, infections, etc. ${ }^{12}$

VTE risk assessment in cancer patients is based on the evaluation of patient-related, tumor-related, and treatmentrelated parameters. ${ }^{13}$ Increased age, comorbidities, and prolonged immobility are relatively common among patients with severe COVID-19 disease. Whether the risk of VTE development in active-cancer patients infected with SARSCoV2 is higher than in COVID-19 patients without malignancies remains to be determined.

\section{Mechanisms of Thrombosis in Cancer and COVID-19 Patients}

The three components of the Virchow's triad are relevant both in cancer and COVID-19 patients. In these diseases, the endothelial wall is damaged, leading to a shift toward a procoagulant phenotype. Stasis of blood is increased due to prolonged hospitalization and immobilization, especially in patients under mechanical ventilation and in those admitted to the ICU. Importantly, proinflammatory cytokines, induced by COVID-19 and cancer, are major contributors to thrombosis development in both clinical scenarios. Indeed, several of these cytokines, reported to be found in patients with SARS-CoV2 infection, are also secreted by tumor and host cells, in the setting of cancer-associated thrombosis (CT). ${ }^{14}$ In this context, tumor necrosis factor- $\alpha$ and interleukin (IL)- 1 can contribute to thrombus formation by inducing tissue factor (TF) and von Willebrand factor (VWF) expression on vascular endothelial cells, increasing the level of the fibrinolysis inhibitor plasminogen activator inhibitor-1 and attenuating anticoagulant effects via downregulation of thrombomodulin expression.

TF has a crucial role in promoting coagulation and angiogenesis in both solid and hematological malignancies. Pulmonary and peripheral endothelial cell injury due to direct viral attack can activate the coagulation system via exposure of TF and other procoagulant pathways. ${ }^{15}$ In a recently published study evaluating patients with COVID-19, endothelial cell involvement across vascular beds of different organs (lung, heart, kidney, liver, and intestine) was demonstrated. ${ }^{16}$ In all cases, viral elements were found within endothelial cells and accumulation of inflammatory cells was observed, with evidence of endothelial and inflammatory cell death. Based on these findings the authors suggest that SARS-CoV-2 infection facilitates the induction of endotheliitis in several organs as a direct consequence of viral involvement and host inflammatory response.

TF is also expressed by mononuclear cells and macrophages in response to proinflammatory cytokines. ${ }^{17}$ In severe inflam- mation, coagulation is mainly dependent on the recruitment of TF-expressing inflammatory monocytes by activated endothelial cells. ${ }^{18}$ Other possible contributors to the induction of TF expression and inflammatory programs in monocytes are oxidized phospholipids. ${ }^{19}$ These molecules which are produced in oxidative stress, have been found in lungs of patients with severe SARS-CoV (another corona virus). ${ }^{20}$

TF-bearing extracellular vesicles (EVs), released from a variety of cells, including tumor and endothelial cells, are known to be involved in $\mathrm{CT} .{ }^{21}$ As EVs are reported to play a role in inflammation and thrombosis, they are likely to take part in the thrombotic process revealed in severe COVID-19 infection.

Likewise, cancer-associated neutrophil extracellular traps (NETs), DNA-associated mesh of neutrophil-derived proteases and histones, may facilitate activation of host cells to promote arterial and thrombotic events. ${ }^{22}$ For instance, NETs can serve as a platform for direct platelet adhesion and aggregation. $^{23}$ Furthermore, NET-associated histones can indirectly augment platelet aggregation by increasing VWF released from activate endothelial cells. Neutrophilia is a prognostic marker in COVID-19 disease and excessive neutrophil infiltrates have been observed in autopsy lung samples of COVID-19 patients. It has been proposed that neutrophilia could also be a source of excess NETs in this setting and that inhibiting NET formation should be a target for therapy in COVID-19. ${ }^{24}$

\section{Management of Cancer Patients Diagnosed with COVID-19}

\section{VTE Prophylaxis}

Recent guidelines emphasize the role of low-molecularweight heparin (LMWH) and direct oral anticoagulants (DOACs) in CT management. ${ }^{25}$ It is crucial that the risk for VTE be evaluated in every acutely ill patients with COVID-19, utilizing the available risk-assessment tools (e.g., the Caprini, IMPROVE, and Padua models). ${ }^{26,27}$ In general, patients with COVID-19 and active cancer should receive pharmacological VTE prophylaxis, unless there are contraindications. Prophylactic daily LMWHs, or twice-daily subcutaneous unfractionated heparin (UFH) are recommended by the World Health Organization interim guidance statement. ${ }^{28}$ It can be argued that due to very high thrombotic risk, patients with active cancer and severe COVID-19 may need intermediate doses of $\mathrm{LMWH}^{29}$ and that monitoring anti-Xa levels could be of help to optimize anticoagulation.

If pharmacological prophylaxis is contraindicated, mechanical VTE prophylaxis (intermittent pneumatic compression) should be considered in immobilized patients. ${ }^{28}$ The role of thromboprophylaxis for nonhospitalized cancer patients with mild COVID-19 is uncertain. However, in patients with active cancer and limited mobility who are quarantined, the use of pharmacologic prophylaxis should be weighed against the risks of bleeding. The role of extended prophylaxis after hospital discharge has not been studied in COVID-19 patients. Nevertheless, for cancer patients, prolonged use of LMWH prophylaxis for up to 6 weeks with monitoring D-dimer levels 
seems reasonable. Although heparin-induced thrombocytopenia (HIT) is uncommon in patients treated with LMWH, fondaparinux could be considered for lowering the risk of HIT in cancer patients with COVID-19.

\section{VTE Treatment}

Therapeutic anticoagulation is the mainstay of VTE treatment in cancer patients with COVID-19. Of note, bleeding risk and renal functions should be taken into consideration. While DOACs are currently increasingly employed in CT, ${ }^{30}$ potential drug-drug interactions with investigational antiCOVID-19 drugs should be taken into account. For example, using lopinavir/ritonavir requires dose adjustments of apixaban and betrixaban, while edoxaban and rivaroxaban should not be co-administered with these drugs. Only dabigatran can be safely used with these agents. Tocilizumab, an IL-6 inhibitor, which may be employed for the treatment of COVID-19-induced cytokine storm, increases expression of CYP3A4; however, no dose adjustments are currently recommended with concomitant use of DOACs at this time. The use of azithromycin (a known P-gp inhibitor) with edoxaban or betrixaban also deserves caution, and dose reduction of these factor Xa inhibitors is recommended. Of note, a further analysis of the drug-drug interaction of apixaban in CT is underway. Taken together, nonoral anticoagulation (e.g., UFH or LMWH) is preferred as it can be temporarily withheld and has no known drug-drug interactions with investigational COVID-19 therapies. ${ }^{31}$ Additional information about potential drug-drug interaction of oral anticoagulants with anti-COVID-19 drugs is available at https://www.covid19druginteractions.org/.

The treatment of VTE in hematological malignancies is challenging as severe thrombocytopenia is common in hematological patients due to bone marrow infiltration by tumor cells, toxicity of chemotherapy or targeted therapies, etc. ${ }^{32}$ Furthermore, in clinical trials of CT, only a minority of patients had hematological malignancies, and usually patients with severe thrombocytopenia were excluded. Recently, a recent guidance from the Scientific and Standardization Committee of the International Society on Thrombosis and Haemostasis on the management of CT patients with thrombocytopenia suggested the following approach. ${ }^{33}$ In brief, in thrombocytopenic (i.e., $<50,000 / \mu \mathrm{L}$ ) patients with high risk for thrombosis progression or recurrence (symptomatic segmental or more proximal PE, proximal DVT, or a history of recurrent/progressive thrombosis), full-dose anticoagulation in conjunction with platelet transfusion should be administered. For patients with lower risk events a dose-modification strategy using $50 \%$ or prophylactic-dose LMWH may be considered. In severe thrombocytopenia (i.e., $<25,000 / \mu \mathrm{L}$ ) anticoagulation should be withheld, although prophylactic doses might be reasonable in patients with a platelet count of $>10,000 / \mu \mathrm{L}$. Inferior vena cava (IVC) filter placement should be considered only in patients with absolute contraindications to anticoagulation. Therefore, retrievable IVC filters may be considered on a caseby-case basis in patients with acute VTE who have severe, prolonged thrombocytopenia or actively bleeding for which anticoagulation with platelet transfusion cannot be achieved.
LMWH is currently the preferred anticoagulant among patients with CT and thrombocytopenia, and DOACS are not recommended.

\section{Conclusion}

Extrapolating the antithrombotic strategy to cancer patients with COVID-19 is challenging due to the paucity of data and the high risk for both thrombosis and bleeding. Rapidly emerging data will hopefully foster management regimens for these critically ill patients.

\section{Conflict of Interest}

Dr. Brenner reports personal fees from Pfizer, LEO Pharma, Sanofi, ROVI Laboratories, and Bayer Pharmaceuticals, outside the submitted work.

\section{References}

1 Middeldorp S, Coppens M, van Haaps TF, et al. Incidence of venous thromboembolism in hospitalized patients with COVID-19. J Thromb Haemost 2020 (e-pub ahead of print). Doi: 10.1111/ jth.14888

2 Helms J, Tacquard C, Severac F, et al; CRICS TRIGGERSEP Group (Clinical Research in Intensive Care and Sepsis Trial Group for Global Evaluation and Research in Sepsis). High risk of thrombosis in patients with severe SARS-CoV-2 infection: a multicenter prospective cohort study. Intensive Care Med 2020;46(06):10891098

3 Lodigiani C, Iapichino G, Carenzo L, et al; Humanitas COVID-19 Task Force. Venous and arterial thromboembolic complications in COVID-19 patients admitted to an academic hospital in Milan, Italy. Thromb Res 2020;191:9-14

4 Llitjos JF, Leclerc M, Chochois C, et al. High incidence of venous thromboembolic events in anticoagulated severe COVID-19 patients. J Thromb Haemost 2020 (e-pub ahead of print). Doi: $10.1111 /$ jth.14869

5 Yu J. Ouyang W, Chua MLK, Xie C. SARS-CoV-2 transmission in patients with cancer at a tertiary care hospital in Wuhan, China. JAMA Oncol 2020 (e-pub ahead of print). Doi: 10.1001/ jamaoncol.2020.0980

6 Liang W, Guan W, Chen R, et al. Cancer patients in SARS-CoV-2 infection: a nationwide analysis in China. Lancet Oncol 2020;21 (03):335-337

7 Kuderer NM, Choueiri TK, Shah DP, et al; COVID-19 and Cancer Consortium. Clinical impact of COVID-19 on patients with cancer (CCC19): a cohort study. Lancet 2020;395(10241): 1907-1918

8 Sharma BK, Flick MJ, Palumbo JS. Cancer-associated thrombosis: a two-way street. Semin Thromb Hemost 2019;45(06):559-568

9 Mahajan A, Brunson A, White R, Wun T. The epidemiology of cancer-associated venous thromboembolism: an update. Semin Thromb Hemost 2019;45(04):321-325

10 Navi BB, Reiner AS, Kamel H, et al. Risk of arterial thromboembolism in patients with cancer. J Am Coll Cardiol 2017;70(08):926-938

11 Adelborg K, Corraini P, Darvalics B, et al. Risk of thromboembolic and bleeding outcomes following hematological cancers: a Danish population-based cohort study. J Thromb Haemost 2019;17 (08):1305-1318

12 Levi M. Management of cancer-associated disseminated intravascular coagulation. Thromb Res 2016;140(Suppl 1):S66-S70

13 Abdol Razak NB, Jones G, Bhandari M, Berndt MC, Metharom P. Cancer-associated thrombosis: an overview of mechanisms, risk factors, and treatment. Cancers (Basel) 2018;10(10): E380 
14 Falanga A, Panova-Noeva M, Russo L. Procoagulant mechanisms in tumour cells. Best Pract Res Clin Haematol 2009;22(01):49-60

15 Clauss M, Gerlach M, Gerlach H, et al. Vascular permeability factor: a tumor-derived polypeptide that induces endothelial cell and monocyte procoagulant activity, and promotes monocyte migration. J Exp Med 1990;172(06):1535-1545

16 Varga Z, Flammer AJ, Steiger P, et al. Endothelial cell infection and endotheliitis in COVID-19. Lancet 2020;395(10234):1417-1418

17 Merad M, Martin JC. Pathological inflammation in patients with COVID-19: a key role for monocytes and macrophages. Nat Rev Immunol 2020;20(06):355-362

18 von Brühl ML, Stark K, Steinhart A, et al. Monocytes, neutrophils, and platelets cooperate to initiate and propagate venous thrombosis in mice in vivo. J Exp Med 2012;209(04):819-835

19 Berliner JA, Watson AD. A role for oxidized phospholipids in atherosclerosis. N Engl J Med 2005;353(01):9-11

20 Imai Y, Kuba K, Neely GG, et al. Identification of oxidative stress and Toll-like receptor 4 signaling as a key pathway of acute lung injury. Cell 2008;133(02):235-249

21 Lacroix R, Vallier L, Bonifay A, et al. Microvesicles and cancer associated thrombosis. Semin Thromb Hemost 2019;45(06):593-603

22 Thålin C, Hisada Y, Lundström S, Mackman N, Wallén H. Neutrophil extracellular traps: villains and targets in arterial, venous, and cancer-associated thrombosis. Arterioscler Thromb Vasc Biol 2019;39(09):1724-1738

23 McDonald B, Davis RP, Kim SJ, et al. Platelets and neutrophil extracellular traps collaborate to promote intravascular coagulation during sepsis in mice. Blood 2017;129(10):1357-1367

24 Barnes BJ, Adrover JM, Baxter-Stoltzfus A, et al. Targeting potential drivers of COVID-19: Neutrophil extracellular traps. J Exp Med 2020;217(06):e20200652

25 Farge D, Frere C, Connors JM, et al; International Initiative on Thrombosis and Cancer (ITAC) advisory panel. 2019 international clinical practice guidelines for the treatment and prophylaxis of venous thromboembolism in patients with cancer. Lancet Oncol 2019;20(10):e566-e581
26 Liu X, Liu C, Chen X, Wu W, Lu G. Comparison between Caprini and Padua risk assessment models for hospitalized medical patients at risk for venous thromboembolism: a retrospective study. Interact Cardiovasc Thorac Surg 2016;23(04):538-543

27 Rosenberg D, Eichorn A, Alarcon M, McCullagh L, McGinn T, Spyropoulos AC. External validation of the risk assessment model of the International Medical Prevention Registry on Venous Thromboembolism (IMPROVE) for medical patients in a tertiary health system. J Am Heart Assoc 2014;3(06):e001152

28 WHO. Clinical management of severe acute respiratory infection (SARI) when COVID-19 disease is suspected. Interim guidance. Available at: https://www.who.int/publications/i/item/clinicalmanagement-of-covid-19. Accessed May 21, 2020

29 Eck RJ, Bult W, Wetterslev J, et al. Intermediate dose low-molecular-weight heparin for thrombosis prophylaxis: systematic review with meta-analysis and trial sequential analysis. Semin Thromb Hemost 2019;45(08):810-824

30 Agnelli G, Becattini C, Meyer G, et al; Caravaggio Investigators. Apixaban for the treatment of venous thromboembolism associated with cancer. N Engl J Med 2020;382(17):1599-1607

31 Bikdeli B, Madhavan MV, Jimenez D, et al. COVID-19 and thrombotic or thromboembolic disease: implications for prevention, antithrombotic therapy, and follow-up. J Am Coll Cardiol 2020;75 (23):2950-2973

32 Napolitano M, Saccullo G, Marietta M, et al; Gruppo Italiano Malattie EMatologiche dell'Adulto (GIMEMA) Working Party on Thrombosis and Hemostasis; Gruppo Italiano Malattie Ematologiche dell'Adulto (GIMEMA) Working Party on Thrombosis and Haemostasis (see Appendix 1). Platelet cut-off for anticoagulant therapy in thrombocytopenic patients with blood cancer and venous thromboembolism: an expert consensus. Blood Transfus 2019;17(03):171-180

33 Samuelson Bannow BT, Lee A, Khorana AA, et al. Management of cancer-associated thrombosis in patients with thrombocytopenia: guidance from the SSC of the ISTH. J Thromb Haemost 2018; 16(06):1246-1249 